\title{
Effect of Confinement Anisotropy on Excitonic Properties in InAs/InP Quantum Dashes
}

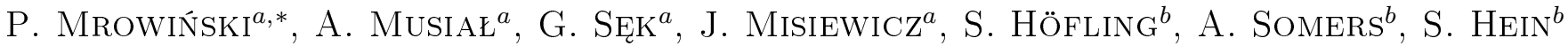 \\ AND A. FORCHEL ${ }^{b}$ \\ ${ }^{a}$ Institute of Physics, Wrocław University of Technology, Wybrzeże Wyspiańskiego 27, 50-370 Wrocław, Poland \\ ${ }^{b}$ Technische Physik, University of Würzburg and Wilhelm-Conrad-Röntgen-Research Center for Complex Material \\ Systems (RCCM), Am Hubland, D-97074 Würzburg, Germany
}

\begin{abstract}
The influence of confinement potential anisotropy on emission properties of strongly elongated single InAs / InGaAlAs/InP quantum dashes has been investigated by polarization-resolved microphotoluminescence spectroscopy at around $1.5 \mu \mathrm{m}$. There have been determined the exciton fine structure splitting, degree of linear polarization of surface emission and biexciton binding energy. The investigated dashes exhibited usually: the exciton anisotropy splitting larger than $100 \mu \mathrm{eV}$, the corresponding biexciton binding energy of about $3 \mathrm{meV}$, and the degree of linear polarization values in the range from $24 \%$ to $55 \%$. Here, we presented a correlation of these parameters for several quantum dashes, which can be attributed either to a change in lateral aspect ratio within the ensemble, or the carrier localization on random fluctuations of the dash confinement potential.
\end{abstract}

DOI: 10.12693 /APhysPolA.124.801

PACS: 71.35.-y, 71.70.Gm, 78.67.Hc, 73.21.-b, 81.07.Ta

\section{Introduction}

Recent development in semiconductor nanotechnology provide many opportunities for realization of completely new concepts in modern information technology, like quantum key distribution [1] or quantum computation [2]. Therefore, it is highly desired to construct an emitter of strictly single photons which could additionally fulfill all the practical requirements, namely well-defined wavelength, polarization state and direction of emission. One of the solutions is to use a quantum-electrodynamics-based system in a solid state matrix, which composes of a single quantum dot in a microcavity $[3,4]$, which has been proven being a good candidate for efficient single photon sources. However, for some of the applications, especially those related to fiber-based optoelectronics and safe data transmission, further development of such sources should be aimed at longer wavelength operation closer to 2nd and 3rd telecommunication windows, i.e. 1.3 and $1.55 \mu \mathrm{m}$. For those, epitaxial nanostructures grown in $\mathrm{InAs} / \mathrm{InP}$ material system seem to be the most suitable [5], where the so called quantum dashes (QDashes) are on one hand typically obtained under common growth conditions of molecular beam epitaxy (MBE), and on the other, has definitely been not explored yet in that respect. The information available in the literature is quite limited and there exist only several reports concerning optical and electronic properties of single InAs/InP quantum dashes emitting in that relevant spectral range [6,7], without any detailed study on the exchange interactions, anisotropy of the confinement potential or the Coulomb correlation effects.

*corresponding author; e-mail: pawel.mrowinski@pwr.wroc.pl
Additional advantage of those structures is related to easily controlled emission range by changing growth parameters, i.e. InAs layer thickness [8]. This has mainly been exploited in optical amplifiers and laser structures $[9,10]$, which had been the original driving forces for the development of such active material. The ensemble of QDashes provides, for instance, a broad and high gain function due to their size and shape fluctuations, and high surface density translating into broad lasing tuneability [11]. More recently, these structures have also been examined in order to describe in detail the anisotropy and spatial character of the confining potential. Polarization-resolved study on the QDash ensemble revealed fluctuations of the individual nanostructure cross-sectional size that can act as trapping centers for carriers [12]. Additionally, several experiments on single QDash-like structures have been reported [13, 14], exhibiting exciton and biexciton cascade recombination close to $1.5 \mu \mathrm{m}$. However, the influence of the confinement anisotropy on the Coulomb correlations and exchange interaction have not been investigated yet for such strongly irregular and anisotropic nanoobjects. Also, whether the size or geometry effects are dominant has not been concluded. This stimulates further and more advanced study of the excitonic complexes. To fill that gap, we focused in this paper on the biexciton binding energy as a probe of the Coulomb correlation effects in QDashes and the exciton fine structure splitting (FSS) which reflects the anisotropy of the exchange interaction.

\section{Investigated structures and experimental setup}

The examined structures were grown by MBE on an InP substrate followed by a $200 \mathrm{~nm}$ thick $\mathrm{In}_{0.53} \mathrm{Ga}_{0.23} \mathrm{Al}_{0.24} \mathrm{As}$ buffer layer latticed-matched to InP, 
which served also as a potential barrier for the dashes. The elongated QDash structures were achieved by deposition of nominally $1.05 \mathrm{~nm}$ thick InAs layer. The sample was then covered with $100 \mathrm{~nm}$ of $\operatorname{In}_{0.53} \mathrm{Ga}_{0.23} \mathrm{Al}_{0.24} \mathrm{As}$ material and finally terminated with $10 \mathrm{~nm}$ of $\mathrm{InP}$ cap. The geometry of all QDashes is driven by a relatively low strain because of slight lattice mismatch of $3.4 \%$ and an anisotropic surface diffusion of atoms. Thus, strongly elongated structures are typically formed in MBE, with principal axes along [110] and [1-10] crystallographic directions. The size of the QDashes can be estimated to be over $100 \mathrm{~nm}$ in length with the lateral aspect ratio (LAR) typically equal to about five, or larger. Their cross-sectional shape can be approximated by a triangle with $3 \mathrm{~nm}$ in height and $16 \mathrm{~nm}$ in width [8] (for the dashes emitting at around $1.5 \mu \mathrm{m}$ ).

All the optical measurements have been performed on a standard microphotoluminescence setup equipped with a microscope objective with a high numerical aperture $N A=0.4$ providing the spatial resolution on the order of a single $\mu \mathrm{m}(2 \mu \mathrm{m}$ diffraction limit for the used excitation). A continuous-wave semiconductor laser of $660 \mathrm{~nm}$ wavelength as an excitation source was used and one-meter focal length monochromator with a liquid nitrogen cooled InGaAs-based CCD served as a detection system. In order to resolve the linear polarization of emission properly, a half-wave plate placed in front of a linear polarizer was rotated. Such a configuration allows us to eliminate the polarization characteristics of the monochromator gratings. The optical characterization was performed on a submicrometer mesa structures etched on the sample surface in order to limit the number of QDashes excited simultaneously (approximately less than a hundred of nanostructures for the smallest mesas of $600 \mathrm{~nm} \times 300 \mathrm{~nm})$.

\section{Experimental results and discussion}

Exemplary low-temperature microphotoluminescence $(\mu \mathrm{PL})$ spectrum of the QDashes is presented in Fig. 1. Due to a lower limit in mesa size and a high surface density of QDashes $\left(10^{11} \mathrm{~cm}^{-2}\right)$, the density of single emission lines is rather high. In presented case the emission covers a range from 1400 to $1475 \mathrm{~nm}$, which corresponds well with the PL emission of the QDash ensemble (blue dashed line). Very often a well-separated single peaks can be observed on the lower-energy side (emission wavelength close to $1500 \mathrm{~nm}$ ). That spectral separation can be optimal for identifying the origin of single emission lines and to assign the excitonic complexes confined within the same QDash.

A relatively simple way to identify the basic excitonic complexes, i.e. exciton and biexciton, is based on the rate equation model, which origins from the kinetics of the carriers (excitons) confined in zero-dimensional objects, characterized by a discrete energy levels with defined lifetimes $[15,16]$. More rigorous method concerns the fine structure splitting of the exciton bright states,

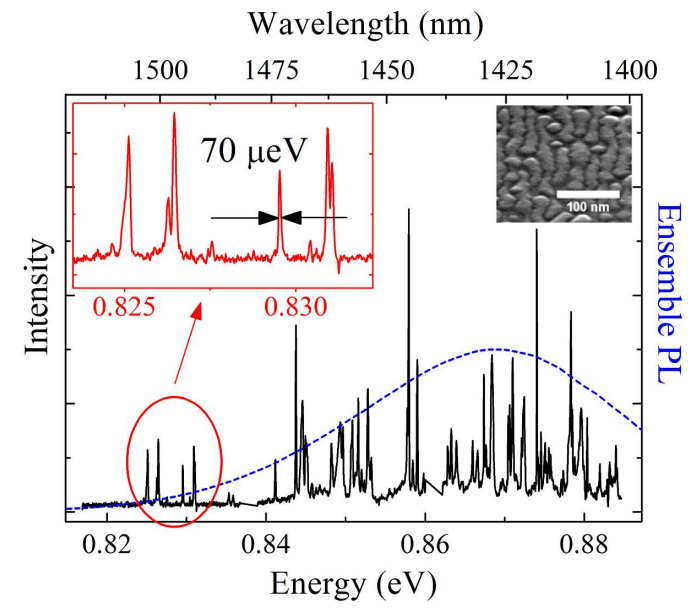

Fig. 1. Microphotoluminescence spectrum taken at $5 \mathrm{~K}$ from the $600 \mathrm{~nm} \times 300 \mathrm{~nm}$ mesa (black solid line), showing well-separated sharp lines around $1500 \mathrm{~nm}$ (left inset) together with emission of the QDash ensemble (blue dashed line). A SEM image of the sample surface is shown in the right inset.

since the anisotropic part of electron-hole exchange interaction lifts the degeneracy and mixes different exciton spin configurations, which results in orthogonal linear polarizations of the emitted photons. What is more, the recombination of biexciton to its final state - non-degenerate exciton - exhibits exactly the same energy splitting. The main advantage of the presented methods is their simplicity from experimental point of view.

In rate equations the only parameter which can be modified during measurements is a generation rate which is proportional to the excitation power density. Thus, by monitoring the peak intensity vs. excitation power, we can distinguish different dependences of excitons and biexcitons, which in the first approximation should depend linearly and quadratically on the excitation power, respectively [16], as long as low excitation is considered. The simulated curves can be matched simultaneously to the experimental data just by tuning the exciton to biexciton radiative lifetime ratio, which is the only fitting parameter.

On the other hand, the fine structure splitting can be observed in polarization-resolved PL when the symmetric oscillations of single emission lines versus polarization angle prove their excitonic and biexcitonic character as well as a common origin.

Figure 2 presents a part of high resolution $(<50 \mu \mathrm{eV})$ $\mu \mathrm{PL}$ spectra taken at low temperature for several excitation powers. For low excitation conditions there are four well-resolved emission lines. One of them, at $852.2 \mathrm{meV}$ is significantly broader than the other, namely its full width at half maximum is $170 \mu \mathrm{eV}$, which suggests a contribution of two closely spaced bright states of the exciton. When the excitation power increases, an additional peak with a similar width appears in the spectrum at $849.5 \mathrm{meV}$. The PL intensity evolution of the higher 


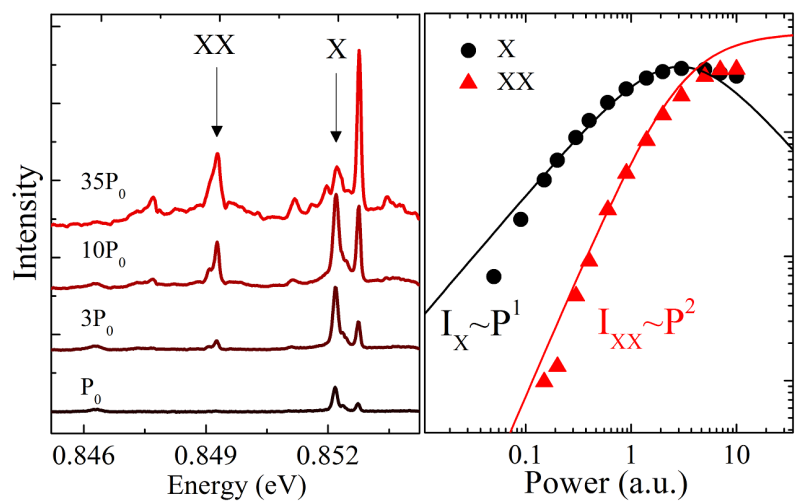

Fig. 2. (left) High-resolution emission spectra for different excitation powers with exciton and biexciton marked by X and XX, (right) integrated PL intensity plotted versus excitation power (symbols) fitted by the rate equation model (solid lines).

energy doublet shows a linear dependence on the excitation power, while the peak on the lower energy side exhibits a quadratic increase.

In Fig. 2 the rate equation model with three levels included was used to fit the experimental data. A very good agreement with theoretical model, especially in the low excitation part, confirms preliminarily the origin of the excitonic and biexcitonic emission from the same QDash.

In order to confirm the abovementioned interpretation, additional linear-polarization-resolved experiment has been performed to examine precisely the exciton fine structure. In Fig. 3 series of spectra for different linear polarizations and fixed excitation power at which biexcitonic emission can be observed is presented. The map shows a 180 degree scan of the spectra for which a symmetric pattern of the fine structure splitting for exciton and biexciton is observed. The angle equal to $0^{\circ}$ reflects the direction along [1-10] crystallographic axis that is parallel to the elongation axis of the QDash. The magnitude of the splitting is $205 \mu \mathrm{eV}$ for both complexes, while the biexciton binding energy is $2.9 \mathrm{meV}$. It is easy to notice a stronger intensity of exciton/biexciton component polarized parallel to [1-10] direction (note that the intensity of the perpendicular component has been magnified by a factor of 2 in Fig. 3 for an easier observation of the splitting). In fact, the degree of linear polarization (DOP) in this case equals to $55 \%$. The DOP was calculated according to standard formula $\Pi=\left(I_{1}-I_{\mathrm{t}}\right) /\left(I_{1}+I_{\mathrm{t}}\right)$, where $I_{1}\left(I_{\mathrm{t}}\right)$ is the emission intensity in the polarization parallel (perpendicular) to the QDash main axis.

Analogical analysis has been performed for more exciton-biexciton cases and a comparison for three representative ones is presented in Table. A general remark concerns a high fine structure splitting (exceeding $100 \mu \mathrm{eV}$ ) and pronounced degree of linear polarization (above 20\%) due to a strong asymmetry of the QDashes. However, the potential asymmetry cannot solely explain

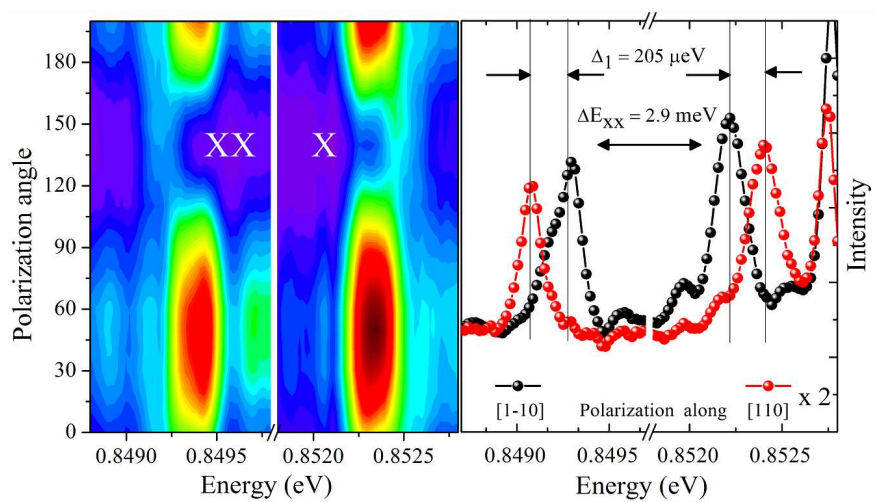

Fig. 3. Polarization-resolved $\mu \mathrm{PL}$ map (left) and orthogonally polarized emission (right) show exciton fine structure splitting of $205 \mu \mathrm{eV}$ and biexciton binding energy of $2.9 \mathrm{meV}$.

such large energy difference between the bright exciton states [17] and additional effects have to be taken into account. We believe that here both strong confinement in the growth direction and substantial in-plane strain anisotropy enlarge the sensitivity of the anisotropic component of the exchange interactions to the LAR of the quantum dash. Namely, even small change in the quantum dash in-plane geometry can result in a noticeable change of the exciton fine structure splitting. A correlation of large energy splitting and degree of polarization with smaller biexciton binding energy $\left(\Delta E_{\mathrm{XX}}\right)$ for the exciton X1 confirms our explanation. According to the mentioned differences in the DOP and FSS for three excitons, we attribute X1 to more elongated QDash. Simultaneously, its $\Delta E_{\mathrm{XX}}$ is lower than for excitons $\mathrm{X} 2$ and X3 which can be explained by the strain-induced piezoelectric field that reduces the Coulomb interactions of electrons and holes because of charge separation [18].

\section{TABLE}

Summary of emission properties of single QDash. (* The results described in detail in the article.)

\begin{tabular}{c|c|c|c}
\hline \hline QDash & $\mathrm{X}^{*}$ & $\mathrm{X} 2$ & $\mathrm{X} 3$ \\
\hline DOP $[\%]$ & $\mathbf{5 5}$ & 25 & 24 \\
FSS $[\mu \mathrm{eV}]$ & $\mathbf{2 0 5}$ & 130 & 150 \\
$\Delta E_{\mathrm{XX}}[\mathrm{meV}]$ & 2.9 & $\mathbf{3 . 6}$ & $\mathbf{3 . 8}$
\end{tabular}

The effect of decreasing $\Delta E_{\mathrm{XX}}$ with increasing size of the nanostructure, and hence also the piezoelectric field, has already been observed in the case of relatively large InAs quantum dots [18, 19]. In our case of nominally the same height of QDashes, the increase of piezoelectric field can origin from a change in the QDash lateral geometry. More elongated QDashes have larger volume and so the piezoelectric effects are stronger leading to enhanced spatial separation of charges which is the cause of the smaller $\Delta E_{\mathrm{XX}}$. Larger values of both the DOP 
and FSS (for X1) are expected in that case due to larger anisotropy of the confining potential. Lower DOP and FSS values (for X2 and X3) can correspond either to less elongated QDashes (probable due to lateral aspect ratio distribution within the ensemble of self-assembled strucures) or additional carrier trapping on potential fluctuations within an individual QDash, both decreasing the effective anisotropy of the confining potential [12].

\section{Conclusions}

Single InAs/InP QDashes have been investigated by polarization-resolved microphotoluminescence. Such system parameters as exciton fine structure splitting, degree of linear polarization as well as the biexciton binding energy have been determined for several dashes showing that emission properties of excitons are strongly influenced by the nanostructure geometry. The observed large values of both FSS and DOP have been attributed to a strong in-plane potential anisotropy, influence of which is further enhanced due to rather strong confinement preserved in the investigated structures. The inter-dash variation of these parameters is caused by changes in the lateral aspect ratio within the QDash ensemble. High FSS and DOP correlate with smaller values of the biexciton binding energy due to stronger piezoelectric effects (separating spatially electrons and holes) for larger nanostructures. Further investigation of the excitonic properties for various confinement strength in the case of strongly anisotropic InAs/InP QDashes together with the proper theoretical modeling of the confinement potential and the excitonic correlations is necessary. This should give an additional insight into the importance and the interplay of other possible effects, i.e. the Coulomb correlation and piezoelectric effects as well as localization of the carriers on confining potential fluctuations.

\section{Acknowledgments}

The authors acknowledge the financial support from the Foundation for Polish Science (FNP) and Deutsche Forschungsgemeinschaft (DFG) - COPERNICUS Award and the National Science Centre of Poland within grants No. 2011/02/A/ST3/00152, 2011/01/B/ ST3/02379 and N N515 496640. The financial support from the Polish Ministry of Science and Higher Education within the investment grant No. 6167/IA/119/2012 is also acknowledged.

\section{References}

[1] C.H. Bennett, G. Brassard, in: Proc. IEEE Int. Conf. on Computers Systems and Signal Processing, Bangalore (India), 1984, p. 175.
[2] E. Knill, R. Laflamme, G.J. Milburn, Nature 409, 46 (2001).

[3] C. Santori, D. Fattal, J. Vuckovic, G.S. Solomon, Y. Yamamoto, New J. Phys. 6, 89 (2004).

[4] T. Heindel, C. Schneider, M. Lermer, S.H. Kwon, T. Braun, S. Reitzenstein, S. Hofling, M. Kamp, A. Forchel, Appl. Phys. Lett. 96, 11107 (2010).

[5] K. Takemoto, M. Takatsu, S. Hirose, N. Yokoyama, Y. Sakuma, T. Usuki, T. Miyazawa, Y. Arakawa, J. Appl. Phys. 101, 081720 (2007).

[6] G. Saint-Girons, N. Chauvin, A. Michon, G. Patriarche, G. Beaudoin, G. Breěmond, C. Bru-Chevallier, I. Sagnes, Appl. Phys. Lett. 88, 133101 (2006).

[7] P. Miska, J. Even, C. Platz, J. Appl. Phys. 95, 1074 (2004).

[8] A. Sauerwald, T. Kümmell, G. Bacher, A. Somers R. Schwertberger, J.P. Reithmaier, A. Forchel, Appl. Phys. Lett. 86, 253112 (2005).

[9] A. Somers, W. Kaiser, J.P. Reithmaier, A. Forchel, M. Gioaninni, I. Montrosset, Appl. Phys. Lett. 89 061107 (2006).

[10] J.P. Reithmaier, G. Eisenstein, A. Forchel, Proc IEEE 95, 1779 (2007).

[11] J.P. Reithmaier, A. Somers, S. Deubert, R. Schwertberger, W. Kaiser, A. Forchel, M. Calligaro, P. Resneau, O. Parillaud, S. Bansropun, M. Krakowski, R. Alizon, D. Hadass, A. Bilenca, H. Dery, V. Mikhelashvili, G. Eisenstein, M. Gioannini, I. Montrosset, T.W. Berg, M. van der Poel, J. Mørk, B. Tromborg, J. Phys. D, Appl. Phys. 38 2088 (2005).

[12] A. Musiał, P. Kaczmarkiewicz, G. Sęk, P. Podemski, P. Machnikowski, J. Misiewicz, S. Hein, S. Höfling, A. Forchel, Phys. Rev. B 85, 035314 (2012).

[13] T. Mensing, L. Worschech, R. Schwertberger, J.P. Reithmaier, A. Forchel, Appl. Phys. Lett. 82, 2799 (2003).

[14] G. Sęk, P. Podemski, A. Musiał, J. Misiewicz, S. Hein, S. Höfling, A. Forchel, J. Appl. Phys. 105, 086104 (2009).

[15] E. Dekel, D. Gershoni, E. Ehrenfreund, J. Garcia P. Petroff, Phys. Rev. B 61, 11009 (2000).

[16] G. Sek, A. Musiał, P. Podemski, J. Misiewicz, J. Appl. Phys. 108, 033507 (2010).

[17] R. Seguin, A. Schliwa, S. Rodt, K. Pötschke, U. Pohl, D. Bimberg, Phys. Rev. Lett. 95, 257402 (2005).

[18] Y. Masumoto, S. Yoshida, M. Ikezawa, S. Tomimoto, Y. Sakuma, Appl. Phys. Lett. 98, 061905 (2011).

[19] N. Chauvin, B. Salem, G. Bremond, G. Guillot, C. Bru-Chevallier, M. Gendry, J. Appl. Phys. 100 , 073702 (2006). 